\title{
Orientación Académica y Profesional a los Menores Extranjeros No Acompañados
}

\author{
Enviado: 6 de febrero de 2020 / Aceptado: 11 de abril de 2020 \\ Publicado: 12 de julio de 2020
}

MIGUEL ÁNGEL MORALES MUÑOZ

Departamento de Métodos de Investigación y Diagnóstico en Educación, Universidad de Granada, España.

M ${ }^{a}$ ELENA PARRA-GONZÁLEZ

Autor de correspondencia.

elenaparra@ugr.es

\section{RESUMEN}

Los Menores Extranjeros No Acompañados (MENA) son menores de otros países que acceden a un país diferente al suyo de forma irregular y sin acompañamiento de un adulto. Se trata de una realidad en nuestra sociedad. Los derechos de estos menores (alimentación, educación, salud, etc.) deben ser los mismos que de cualquier menor español. El siguiente trabajo de investigación, en relación a la educación de estos menores, pone de manifiesto la importancia de la orientación educativa y laboral como un recurso básico para la toma de decisiones y para poder adaptarse a los diferentes contextos donde se desenvolverán a lo largo de sus vidas. Para ello, se realiza una entrevista a los menores, se extrae información real que permite establecer una serie de necesidades educativas y laborales y se planifica una pequeña propuesta de mejora. Finalmente se obtienen algunas conclusiones centradas en la precariedad de los trabajos que han realiza-

\section{ABSTRACT}

Academic and Professional Guidance to the Unaccompanied Foreign Minors

The Unaccompanied Foreign Minors (MENA) are minors from other countries who access a country different from their own in an irregular manner and without the accompaniment of an adult. This is a fact in our society. The rights of these minors (food, education, health, etc.) should be the same as the Spanish minors have.

The following research work, with respect to education, highlights the importance of educational and employment guidance as a basic resource for decision making and to be able to adapt to the different contexts where they will develop throughout their lives. To do this, an interview is conducted with minors, real information is extracted that allows establishing a series of educational and labor needs and a small improvement proposal is planned. Finally, some conclusions were obtained fo- 
do, en la carencia de titulaciones que garanticen un futuro laboral digno y en que lo que más les urge no es otra cosa que acceder a un empleo cualquiera con el objetivo de regularizar su situación legal en el país.

Palabras Clave: Menores extranjeros no acompañados, orientación educativa, orientación profesional. cusing on the precasiousness of the work they have done, on the lack of cualifications that guarantee a decent work future and that what is most urgent for them is nothing more than accesing any job with the objective of regularizing your legal situation in the country.

Keywords: Foreign Unaccompanied Minors, educational guidance, professional guidance.

\section{Introducción}

El fenómeno migratorio está adquiriendo importante protagonismo y podemos apreciarlo a diario a través de los diferentes medios de comunicación. No se trata de un fenómeno localizado en determinados países, sino que se da a nivel mundial. Según un informe del Ministerio del Interior, en lo que llevamos de año 2019, han entrado aproximadamente unas 7.398 personas de manera irregular en nuestro país por medio de embarcaciones, donde también se contabilizan menores sin acompañamiento. Para estos menores y para todos en general, la educación supondrá una herramienta básica para su supervivencia en el país y su futura integración social.

En el caso de los menores, se llevan a cabo escolarizaciones en el sistema educativo reglado una vez alcanzadas determinadas pautas y requerimientos básicos. Lo que nos ocupa en este trabajo, está referido a los menores extranjeros no acompañados (MENA) de la Ciudad Autónoma de Ceuta (España).

Una primera aproximación al concepto de MENA (Menores Extranjeros No Acompañados), se basa en la ofrecida por UNICEF (2009), refiriéndose a aquellos niños/as y adolescentes menores de 18 años que están fuera del país de origen, estando a su vez separados de sus padres o cualquier otra persona que los tuviera a su cargo o disponga de su tutela. En el momento en que un menor cruza una frontera de un país no perteneciente a la Unión Europea a otro europeo sin acompañamiento o presencia de un adulto responsable, se le considera Menor Extranjero No Acompañado y automáticamente la tutela legal de estos menores pasa a ser parte de las autoridades europeas hasta que cumplen los dieciocho años de edad (Mendoza y Morgade, 2018). Arrieta, Etxebarria, Garmendia y Muria (2012), concluyen en que por mayoría son de género masculino, de entre trece y diecisiete años de edad, de nacionalidad marroquí o saharaui, y que proceden de familias con escasos recursos, tanto económicos como educativos. Además, sus necesidades psicológicas, según Manzani y Arnoso (2014) se manifiestan debido a que atraviesan por un proceso de adaptación difícil desde que deciden abandonar su país.

En base a esto, la ORDEN EDU/849/2010, de 18 de marzo, por la que se regula la ordenación del alumnado con necesidad de apoyo educativo y se regulan los servicios de orientación educativa en el 
ámbito de gestión del Ministerio de Educación, en las ciudades de Ceuta y Melilla, es la encargada de organizar la escolarización de este tipo de alumnos en el sistema educativo reglado.

Para hablar de orientación de manera holística, destacamos la definición dada por el Consejo de Europa (Chisvert, 2014), la cual se centra en el acompañamiento en los ámbitos profesionales, sociales y personales, independientemente de la edad que se tenga, para que las personas puedan decidir en materia de educación, empleo, atendiendo en todo momento a sus capacidades y competencias. Bisquerra (2006) añade otras cuestiones que aluden al desarrollo integral de la persona y por tanto de su personalidad a lo largo de toda la vida. Según García (2016), el papel de la orientación ha sido siempre el de atender y por lo tanto reducir las desigualdades que existan en los individuos, ya sean de índole personal o social.

La orientación educativa surge en el siglo XX, debido a los continuos cambios relacionados con la tecnología industrial, lo que dio paso a nuevas formas de formación (Saúl, López y Bermejo, 2009). No se trata de un fenómeno nuevo, sino que lleva presente bastante tiempo, aunque no quizá de la manera sistematizada en la que se concibe en la actualidad, pero sí manteniendo la idea de ayudar a los que la recibían. Actualmente, podríamos referirnos a la orientación educativa como un sinónimo de toma de conciencia dentro del proceso de enseñanza-aprendizaje de un individuo y que fomenta y desarrolla la autonomía del mismo sin necesidad de terceros con respecto a sus futuras decisiones en el ámbito escolar y formativo en general (Martínez, 2013).

Por otra vertiente, pero siguiendo en la misma dirección, Álvarez (citado en Molina, 2004), establece que la orientación profesional se basa en un proceso que ayuda y guía que dirige a las personas en cualquier dimensión de su vida (formativa, profesional, de tiempo libre) buscando prepararlas para la vida adulta. Así pues, debe pretender una adaptación íntegra del sujeto en las mejores condiciones y fomentando aptitudes físicas y psicológicas orientadas a la actividad profesional en concreto (Tintaya, 2016).

Como no puede ser de otra forma, es importante resaltar el marco legislativo donde se apoyan todas las acciones referidas a escolarización y orientación educativa y profesional de los MENA. En la Constitución Española, Artículo 39, capítulo 4, afirma que "Los niños gozarán de la protección prevista en los acuerdos internacionales que velan por sus derechos" (art. $39 \mathrm{CE}$ ). En el marco internacional, encontramos la Declaración de los Derechos del Niño (1989), donde se manifiesta que "el niño por su falta de madurez física y mental, necesita protección y cuidados especiales, incluso la debida protección legal, tanto antes como después del nacimiento” (p. 1).

En materia de inmigración, encontramos la Ley Orgánica 4/2000, de 11 de enero, sobre derechos y libertades de los extranjeros en España y su integración social. Se configuran medidas específicas para 
favorecer la integración de los menores inmigrantes al sistema educativo y así cumplir con la escolarización en edad obligatoria.

La LOMCE, defiende la equidad educativa del alumnado, la igualdad de derechos, la no discriminación y la inclusión educativa, de la misma forma la Orden EDU/849/2010 citada anteriormente, pone de manifiesto la necesidad de atención a los alumnos con necesidades de apoyo educativo, entre los que se incluyen los que se encuentran en situación de desventaja socioeducativa con respecto a los demás, donde se incluyen a los MENA que ocupan este trabajo.

Como hemos visto, nuestra legislación en materia educativa es acogedora e inclusiva con el alumnado diverso, entendido esto como la propia diversidad, pero esta intención no puede verse afectada por factores que hacen que la propia integración de estos alumnos en el sistema educativo sea estigmatizada o simplemente insuficiente. El mundo educativo está en constante cambio y dinamismo social, y como apunta Leiva (2017), no es bastante visibilizar la diferencia para llevar a cabo propuestas pedagógicas interculturales, sino que es necesario, tal como vivimos inmersos en la diversidad cultural a nivel no solo en España ni en Europa, sino en el mundo, desarrollar verdaderos mecanismos educativos interculturales que atiendan las relaciones interpersonales haciendo especial hincapié en la dimensión emocional, en el desarrollo del talento y la creatividad de las personas que participan en el proceso educativo de los alumnos y alumnas, incluyendo a estos, a los docentes y a las familias.

El objetivo general de este trabajo es analizar y conocer la situación de los MENA con respecto a su situación educativa y profesional, así como definir una propuesta de intervención para mejorar su situación actual.

Este objetivo general, se fragmenta en los siguientes objetivos específicos:

- Sintetizar información sobre la orientación en general, educativa y profesional.

- Dotar de herramientas de búsqueda de empleo a los menores.

- Consultar la legislación relativa a los menores y su derecho a la escolarización dentro del sistema educativo español.

- Conocer la situación actual de los MENA del Centro de Acogida de la Esperanza con respecto a las dimensiones educativa y profesional.

- Conocer los intereses y motivación de los MENA. 


\section{Material y método}

La metodología de esta investigación ha consistido en la realización de un estudio de corte cualitativo, pues permite comprender un problema a estudiar de manera general (López, 2002) al a vez que se concibe un proceso flexible y abierto, permitiendo de esta forma el ajuste gradualmente con respecto al objeto de estudio en paralelo al desarrollo del mismo (Salgado, 2007). En cuanto a la técnica de recogida de información, se trata de la entrevista, pues según Moral (2006), esta herramienta permite una comprensión del problema más profunda y una eficaz triangulación de los aspectos que intervienen en el mismo.

Del mismo modo se ha diseñado una propuesta de intervención con el objetivo de paliar o reforzar las carencias y necesidades detectadas con respecto al colectivo dentro del ámbito personal, académico y profesional.

Los participantes que han intervenido son 18 menores del centro de acogida, con edades que oscilan entre los 14 y los 17 años. Todos ellos son residentes del centro y acuden a clases de español que se imparten dentro del mismo.

La entrevista, se ha diseñado a través de un guión de entrevista estructurada, pues como apuntan algunos autores como por ejemplo Ballestar, Orte y Oliver (2003), las entrevistas en la investigación social permiten saber cómo son las cosas en realidad exentas de posibles subjetividades del investigador. Se ha configurado atendiendo a los autores consultados a lo largo del marco teórico de este trabajo.

La entrevista se compone de diez cuestiones relacionadas con aspectos sociodemográficos como la edad, el origen, el tiempo que lleva en la ciudad y si poseen alguna habilidad de manera natural o experiencial, siempre desde el anonimato. Dichas cuestiones están divididas en tres dimensiones; personal, educativa y una tercera referida a la motivación por llegar a donde se proponen. A modo gráfico, se presenta las preguntas realizadas a los menores, cada una con ejemplos o posibilidades de respuestas que ayudan a los mismos a contextualiza cada pregunta.

Cabe mencionar, que la participación de los usuarios ha sido de manera voluntaria y tras obtener autorización para ello. Así mismo, hemos contado con dos usuarios, los cuales son de máxima confianza para ellos, que dominan mejor el castellano para que hicieran de intérprete entre el entrevistador y los menores (ya que su lengua materna es el árabe). Esto ha permitido una recogida de información fiel y contextualizada, pues en su mayoría, no conocen la lengua castellana.

Las respuestas obtenidas se iban anotando a mano y después se han pasado a ordenador para ser introducidas y clasificadas en el programa de análisis de datos cualitativos NVIVO. 
Tabla 1. Instrumento de recogida de información.

\section{Preguntas Entrevista}

\section{Preguntas personales}

1. ¿Qué edad tienes?

2. ¿De dónde eres?

3. ¿Cuánto tiempo llevas en Ceuta?

Autor: Consejo de Europa, (Chisvert, 2014, p. 10)

“...partiendo de sus capacidades, competencias y sean capaces, además, de gestionar todos estos elementos a lo largo de su vida".

4. ¿Posees algún tipo de habilidad de manera natural o experiencia profesional de algún tipo? (pintar, idiomas, cocinar, cantar, bailar, mecánico, albañil...)

5. ¿Posees alguna titulación? (Graduado escolar o equivalente en país de origen, secundaria o equivalente...)

6. ¿Conoces la oferta educativa del Sistema Educativo que se ajusta a tu situación actual? (PCPI, ESO, FP de grado Medio, Superior...).

7. Conociendo las diferentes opciones, ¿Qué te gustaría estudiar? (educación, sanidad, artes, administración...).

Autor: Bisquerra 2006, p. 10.

“...debe llegar a todas las personas, en todos sus aspectos de desarrollo personal y durante todo el ciclo vital”.

8. ¿Cómo te imaginas en un futuro cercano? (estudiando algo que te guste, trabajando en algo que ya conoces o algo nuevo para ti...).

9. ¿Crees que puedes llegar a ser como imaginas? (motivación para llegar donde desea).

10. ¿Sabes qué debes hacer para llegar ahí? (¿Qué tienes que estudiar, qué requisitos, educativos, experienciales, son necesarios para llegar donde quieres...). 


\section{Resultados}

Los datos analizados con el programa NVIVO se presentan de la siguiente forma:

Atendiendo a la ciudad de origen, el $85 \%$ de los menores son de Castillejos, un pueblo cercano a la frontera con España, excepto algunos que proceden de Tetuán o Chef Chaouen, ciudades situadas más adentradas en Marruecos.

En lo relacionado con las habilidades encontramos deportes como el fútbol, valleyball o atletismo, idioma, rap, teatro etc. Y en cuanto a la experiencia profesional, trabajos como mecánico en talleres de coches, pintores, peluquería, tapicería, etc.

Más del 70\% de los usuarios no posee titulación alguna, aunque algunos poseen algún diploma sobre electricidad, peluquería o uno de ellos, un título equivalente a la ESO en España. La totalidad de los menores desconocen la oferta educativa del sistema educativo español.

La vocación educativa y laboral, se declina en su mayoría hacia oficios como policía, traductores o cocineros.

La idea que poseen de ellos mismos en un futuro cercano se centra en estar estudiando y/o trabajando en la península en sus casas de propiedad, teniendo posibilidades para formar una familia y ahorrar con el fin de ayudar a su familia en Marruecos. Algunos exponían: "solo quiero vivir de manera normal, sin problemas y teniendo para comer y poder vivir con dignidad y ayudar a mi familia".

El 100\% de los usuarios, desconoce la existencia de herramientas para la búsqueda activa de empleo, como el currículum vitae, la carta de presentación, etc.

Tabla 2. Resultados

\begin{tabular}{|l|c|c|}
\hline & SI & NO \\
\hline País de Origen (Marruecos) & $100 \%$ & \\
\hline Posee alguna titulación & $30 \%$ & $70 \%$ \\
\hline Desconocen oferta educativa española & $100 \%$ & \\
\hline Desconocen herramientas de búsqueda de empleo & $100 \%$ & \\
\hline
\end{tabular}

La motivación para estudiar lo que quieren o trabajar para llegar a ser tal como se imaginan en un futuro cercano es muy alta, pues necesitan experimentar cuanto antes un cambio en sus 
circunstancias de vida actuales. Uno de ellos decía: “Estoy cansado de que siempre piensen de mí que soy un ladrón y voy a molestar a los demás porque no soy de España”.

\section{Discusión}

Atendiendo a los resultados obtenidos, podemos establecer una serie de aspectos coincidentes en relación con otros autores o instituciones que han estudiado esta misma temática.

En este sentido la Asociación Pro Derechos Humanos de Andalucía (2006), concluye en un estudio realizado con el colectivo de MENA, que estos menores han tenido una experiencia profesional centrada en trabajos precarios y de baja cualificación desde muy temprana edad debido a la interrupción de sus estudios por problemas familiares, al igual que los entrevistados en este trabajo, los cuales, señalan que la mayor parte del trabajo que han hecho ha estado basado en trabajos manuales como pintores, mecánicos, tapiceros, en edades comprendidas entre los 13 y los 14 años.

Es evidente, que debido a la situación socioeconómica de estos menores, no están en posesión de titulaciones académicas básicas o medias que les permita acceder a un empleo cualificado, por ello, apoyamos nuestro trabajo en Etxebarria, Garmendia y Murua (2012), donde especifican que los menores deben dejar sus estudios para atender a carencias familiares por lo que carecen de formación académica adecuada para encontrar un trabajo con garantías en el país de destino.

La vocación laboral de los usuarios se inclina hacia policía, peluquería, soldado, pero por otro lado, la totalidad de los menores manifiestan que trabajarían donde fuera necesario, tal como corrobora el Observatorio de la Inmigración de Tenerife (2010) en su investigación realizada a los MENA en esta localidad exponiendo que mientras que las instituciones educativas se encargan de diseñar itinerarios personalizados, los menores poseen un proyecto migratorio principalmente centrado en la inmediata incorporación a cualquier trabajo para regularizar su situación legal cuanto antes dentro del país, aspecto que también es respaldado en una investigación realizada por Bravo y Santos (2017), donde recogen en las entrevistas llevadas a cabo con los menores, que su mayor aspiración en su proyecto de migratorio es lograr tener un trabajo y alcanzar una mejor vida en Europa.

\section{Conclusiones}

Como conclusiones finales, podemos determinar que estos menores:

- Carecen de titulaciones educativas oficiales que les sirva de puente a un empleo digno o acorde a sus preferencias. 
- No conocen la oferta educativa del sistema educativo español.

- No conocen herramientas para la búsqueda de empleo.

\section{Limitación y prospectiva}

La principal limitación del estudio es la falta de tiempo para haber planteado una intervención a la luz de los resultados obtenidos y llevarla a cabo, analizando posteriormente las posibles diferencias entre los resultados. En un futuro próximo se pretende avanzar en este sentido en esta investigación.

\section{Referencias}

Arrieta,E., Etxebarria, F., Garmendia, J. y Murua, H. (2012). Menores Inmigrantes No Acompañados (MENA) en Euskadi y Aquitania: elaboración y puesta en marcha de un Plan de Formación para Educadores/as y Responsables. Revista de Educación Social, 15.

Asociación Pro Derechos Humanos de Andalucía. (2006). Entre la represión y la protección. Menores Extranjeros No Acompañados en Andalucía. Recuperado de: http://www.mediadiversity.org/en/additional-files/documents/b-studies-reports/Unaccompanied\%20Foreign\%20Minors\%20in\%20Andalucia\%20\%5BE S\%5D.pdf

Ballester,L.,Orte, C.y Oliver,J.L.(2003). Análisis cualitativo de entrevistas.Nómadas, 18. 140-149.Recuperado de: http://www.redalyc.org/service/redalyc/downloadPdf/1051/105117890013/1

Bisquerra,R.(2006).Orientación psicopedagógica y educación emocional.Estudios sobre Educación, 11, 9-25. Recuperado de: http://www.ub.edu/grop/wp-content/uploads/2014/03/ Orientaci\%C3\%B3n-psicopedag\%C3\%B3gica- y-educaci\%C3\%B3n-emocional.pdf

Bravo, A y Santos, I. (2017). Menores Extranjeros No Acompañados en España: Necesidades y Modelos de intervención. Pychosocial Intervention, 26(1), 55-62. DOI: http://dx.doi. org/10.1016/j.psi.2015.12.001.

Chisvert, Ma José. (2014). Revisión del Desarrollo de la Orientación Sociolaboral como Política Activa de Empleo. Revista Española de Orientación y Psicopedagogía, 25(1), 8-24. DOI: https://doi.org/10.5944/reop.vol.25.num.1.2014.12010 
Constitución Española. Boletín Oficial del Estado, 29 de diciembre de 1978, núm. 311, pp. 29313 a 29424.

España. Orden EDU/849/2010, de 18 de marzo, por la que se regula la ordenación del alumnado con necesidad de apoyo educativo y se regulan los servicios de orientación educativa en el ámbito de gestión del Ministerio de Educación, en las ciudades de Ceuta y Melilla. Boletín Oficial del Estado, 6 de abril de 2010, núm. 83, pp. 31350 a 31355.

García, L., (2016). El desarrollo de la orientación educativa en el aprendizaje-servicio. Un estudio de caso en instituto de educación secundaria de la comunidad de Madrid (Tesis doctoral). Universidad Autónoma de Madrid, Madrid. Recuperado de https://repositorio.uam. es/bitstream/handle/10486/674897/garcia_lopez_maria_lourdes.pdf

Leiva, J. (2017). La educación intercultural hoy: reflexiones y perspectivas pedagógicas, Revista Complutense de Educación, 28 (1), 29-43. DOI: https://doi.org/10.5209/rev RCED.2017.v28.n1.48589

Ley Orgánica 4/2000, de 11 de enero, sobre derechos y libertades de los extranjeros en España y su integración social, 10, de 12 de enero de 2000, 1 a 50. Recuperado de https://www.boe. es/buscar/pdf/2000/BOE- A-2000-544-consolidado.pdf

López, F. (2002). El análisis del contexto como método de investigación. Revista de educación, 4. 167-179. Recuperado de: http://rabida.uhu.es/dspace/bitstream/handle/10272/1912/ b15150434.pdf?seq

Manzani, L., y Arnoso, M. (2014). Bienestar psicosocial en menores y jóvenes extranjeros sin referente familiar adulto: factores de riesgo y protección. Norte de Salud Mental, 49(12). 33-45. Recuperado de: https://dialnet.unirioja.es/descarga/articulo/4830464.pdf

Martínez, A., (2013). La orientación como actividad educativa y vocacional en los itinerarios curriculares del alumnado de bachiller y formación profesional y su inclusión en el mercado laboral (Tesis doctoral). Universidad de Granada, Granada. Recuperado de http:// digibug.ugr.es/handle/10481/30837

Mendoza Pérez, K., \& Morgade Salgado, M. (2018). Doing Masculinity: The "Look” of Unaccompanied Male Migrant Teenagers from the Maghreb. Men and Masculinities, 21(3), 405. Recuperado de: https://doi.org/10.1177/1097184X17748169

Ministerio del Interior (2019). Inmigración irregular en 2019. Recuperado de: http://www.interior. 
gob.es/documents/10180/10275911/10 informe quin cenal acumulado 01-01 al 0206-2019.pdf/3b69e283-671e-4fc7-a237-90983c58119f

Molina Contreras, D.L. (2004). Concepto de Orientación Educativa: Diversidad y Aproximación. Revista Iberoamericana de Educación, 35(1), 1-22. Recuperado de https://rieoei.org/ $\underline{\text { RIE/article/view/2924/3848 }}$

Moral, C., (2006). Criterios de evaluación en la investigación cualitativa actual. Revista de investigación educativa, 1(24). 147-164. Recuperado de: https://digitum.um.es/digitum/bitstream/10201/45190/1/Criterios\%20de\% 20validez\%20en\%20la\%20investigacion $\% 20$ cualitativa\%20de\%20la\%20 objetividad\%20a\%20la\%20solidaridad.pdf

Observatorio de la Inmigración de Tenerife. (2010). La inmigración irregular en Tenerife: informes temáticos. ISBN: 978-84-692-8901-3. Recuperado de: https://issuu.com/obitenpublicaciones/docs/investigacion_menores

ONU: Asamblea General,Convención sobre los Derechos del Niño, 20 Noviembre 1989,United Nations, Treaty Series, vol. 1577, p. 3. Recuperado de: https://www.refworld.org.es/ docid/50ac92492.html

Salgado, A.C. (2007). Investigación cualitativa: diseños, evaluación del rigor metodológico y retos. LIBERABIT, 13, 71-78. Recuperado de: http://www.scielo.org.pe/pdf/liber/v13n13/ a09v13n13.pdf

Saúl, L., López, M., y G. Bermejo, B. (2009). La orientación educativa en las universidades españolas: de la orientación laboral y vocacional a la atención psicológica. Acción Psicológica, 6(1), 7-15. Recuperado de: http://portal.uned.es/pls/portal/docs/PAGE/UNED MAIN/LAUNIVERSIDA D/UBICACIONES/02/DOCENTE/LUIS ANGEL SAUL GUTIERREZ/PU BLICACIONES/2009 LA-ORIENTACI-EDUCATIVA-UNIVERSIDADES- ESPA.PDF

Tintaya, P. (2016). Orientación profesional y satisfacción vocacional. Revista de Investigación Psicologica,15, pp. 48. Recuperado de http://www.scielo.org.bo/scielo.php?script=sci arttext\&pid=S2223303220 16000100004\&lng=es\&tlng=es.

UNICEF, 2009. "Ni ilegales ni invisibles". Realidad jurídica y social de los Menores Extranjeros en España. Informe 2009. Recuperado de https://www.unicef.es/sites/unicef.es/files/informe infancia inmigrante UNICEF CGAE 2009.pdf 